\title{
A Importância da Autópsia na Morte Neonatal Precoce em Portugal
}

\author{
The Importance of Autopsy in Early Neonatal Death in \\ Portugal
}

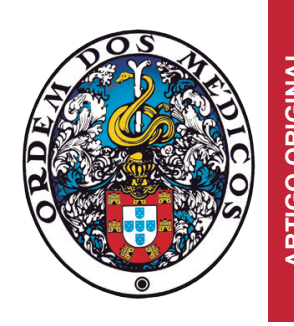

\author{
Marlene MIRANDA $\triangle^{1}$, Sandra COSTA ${ }^{1,2}$, Henrique SOARES ${ }^{1,2}$, Joselina BARBOSA ${ }^{1,3,4}$, Filipa FLOR-DE-LIMA ${ }^{1,2}$, \\ Manuela RODRIGUES ${ }^{2}$, Hercília GUIMARÃES $1,2,4$ \\ Acta Med Port 2020 Dec;33(12):811-818 - https://doi.org/10.20344/amp.12182
}

\section{RESUMO}

Introdução: O período neonatal precoce é o mais crítico para a vida do recém-nascido. A autópsia é importante para compreender a causa de morte e conhecer outros diagnósticos não identificados clinicamente. No entanto, a taxa de autópsia neonatal está a diminuir em todo o mundo. Este estudo pretende caracterizar a morte neonatal precoce e a importância clínica da autópsia, avaliando a concordância entre o diagnóstico clínico e o anatomopatológico.

Material e Métodos: Estudo retrospetivo dos processos clínicos de todos recém-nascidos admitidos numa unidade de Cuidados Intensivos Neonatais de nível III em Portugal e que faleceram durante a primeira semana de vida em 10 anos consecutivos (2008 2017). Para classificar a concordância encontrada entre os diagnósticos clínicos e anatomopatológicos foi usada a classificação de Goldman modificada.

Resultados: Na primeira semana de vida faleceram 76 recém-nascidos. As principais causas de morte foram complicações relacionadas com prematuridade e anomalias congénitas. A autópsia foi realizada em $50(65,8 \%)$ recém-nascidos. Achados adicionais foram encontrados em $62 \%$ dos casos, sendo em $12 \%$ achados com implicações importantes no aconselhamento genético de futuras gestações. A concordância entre os achados clínicos e anatomopatológicos foi de $38 \%$ dos casos.

Discussão: A autópsia foi realizada com maior frequência em recém-nascidos com maior idade gestacional. O número de diagnósticos adicionais encontrados na autópsia, incluindo diagnósticos com implicações para aconselhamento genético, confirmam a importância da sua realização.

Conclusão: A autópsia deve ser proposta a todos os pais após a morte neonatal precoce, dada a sua importância no esclarecimento da causa de morte.

Palavras-chave: Autópsia; Causas de Morte; Mortalidade Infantil; Recém-Nascido; Unidade de Cuidados Intensivos Neonatais

\section{ABSTRACT}

Introduction: The early neonatal period is the most critical for the newborn's life. The autopsy is important to understand the cause of death, and find other diagnoses not clinically identified. However, the rate of neonatal autopsy is declining worldwide. This study aims to characterize early neonatal death and the clinical importance of the autopsy, evaluating the concordance between clinical and pathological diagnosis.

Material and Methods: Retrospective study of the clinical records of all neonates admitted to a level III Neonatal Intensive Care unit in Portugal who died during the first week of life in 10 consecutive years $(2008-2017)$. In order to classify the concordance found between clinical and pathological diagnoses, the modified Goldman classification was used.

Results: During the first week of life, 76 newborns died. The main causes of death were complications related with prematurity and congenital malformations. The autopsy was performed in 50 newborns. Additional findings were found in $62 \%$ of the cases, and in $12 \%$ findings with important implications for genetic counseling of future pregnancies. There was concordance between the clinical and pathological findings in $38 \%$ of cases.

Discussion: An autopsy was performed more frequently in newborns with greater gestational age. The number of additional diagnoses found at autopsy, including diagnoses with implications for genetic counseling, confirm the importance of performing them.

Conclusion: An autopsy should be proposed to all parents after early neonatal death, given its importance in clarifying the cause of death.

Keywords: Autopsy; Causes of Death; Infant Mortality; Infant, Newborn; Neonatal Intensive Care Unit

\section{INTRODUÇÃO}

A morte neonatal precoce é definida como a morte de recém-nascidos entre os zero e sete dias de vida. Mundialmente, $36 \%$ das mortes em recém-nascidos ocorreram nas primeiras 24 horas de vida e cerca $75 \%$ de todas as mortes neonatais ocorreram na primeira semana. ${ }^{1,2}$ Nos recém-nascidos, o diagnóstico clínico é muitas vezes difícil, uma vez que as manifestações de muitas doenças são inespecíficas $^{3}$ e o estado crítico dos recém-nascidos muitas vezes não permite o uso atempado de meios complementares de diagnóstico que permitiriam esclarecer a situação clínica.

O estudo anatomopatológico tem sido muito importante, contribuindo significativamente para o conhecimento clínico. ${ }^{4}$ Permite identificar achados adicionais não suspeitos clinicamente, avaliar a precisão do diagnóstico e a eficácia e segurança de intervenções diagnósticas e terapêuticas, contribuindo para a educação médica e proporcionando

1. Faculdade de Medicina. Universidade do Porto. Porto. Portugal.

2. Serviço de Neonatologia. Centro Materno Pediátrico. Centro Hospitalar Universitário São João. Porto. Portugal.

3. Departamento de Ciências da Saúde Pública e Forenses e Educação Médica. Faculdade de Medicina. Universidade do Porto. Porto. Portugal.

4. Unidade de Investigação e Desenvolvimento Cardiovascular. Faculdade de Medicina. Universidade do Porto. Porto. Portugal.

$\square$ Autor correspondente: Marlene Miranda. Imarlenemirandas@gmail.com

Recebido: 16 de abril de 2019 - Aceite: 08 de janeiro de 2020 | Copyright @ Ordem dos Médicos 2020 
dados estatísticos mais corretos de saúde pública. ${ }^{4} \mathrm{~A}$ autópsia neonatal tem particular importância para os pais, uma vez que o esclarecimento da causa de morte pode contribuir significativamente para ajudar no processo de luto e pode fornecer informações sobre condições relevantes para aconselhamento genético de futuras gestações. ${ }^{5,6}$ Há autores que referem que os pais que recusaram a realização de exame necrópsico lamentaram a sua decisão mais frequentemente em comparação com os pais que aceitaram a sua realização. ${ }^{5}$

Embora a literatura descreva muitas vantagens na realização de exame anatomopatológico, o declínio da taxa de autópsia em recém-nascidos internados em unidade de cuidados intensivos neonatais (UCIN) tem sido registado em diferentes países. Este facto pode ser explicado pela dificuldade dos clínicos em enfrentar os pais bem como em Ihes explicar adequadamente o valor do exame necrópsico. As crenças religiosas ou culturais, o atraso do funeral e o medo da desfiguração do recém-nascido também contribuem para a dificuldade em obter o consentimento para a sua realização. ${ }^{3} \mathrm{O}$ desenvolvimento das técnicas de diagnóstico, que permitem aos clínicos ter mais certezas no diagnóstico clínico também pode explicar esta diminuição. ${ }^{4,7,8}$

Métodos não invasivos de estudo post-mortem têm sido estudados como alternativa à autópsia. A ressonância magnética post-mortem tem sido uma das mais estudadas, sendo mais aceite pelos pais por ser menos invasiva. No entanto, há que ter em consideração os limites de resolução na deteção de certos tipos de lesões a ela inerentes. ${ }^{9}$ Um estudo que comparou os achados adicionais na autópsia com a sua possibilidade de deteção por ressonância magnética demonstrou que mais de $55 \%$ dos achados adicionais encontrados na autópsia provavelmente não seriam detetados na ressonância magnética post-mortem. ${ }^{3}$
A biópsia por agulha percutânea também tem sido estudada como uma alternativa por ser minimamente invasiva e por essa razão mais aceite pelos pais, no entanto, tem uma alta taxa de falsos negativos, uma vez que, uma lesão localizada dentro do órgão pode não ser detetada. ${ }^{10}$

Este estudo tem como objetivo caracterizar as causas de morte neonatal precoce e avaliar a importância da autópsia neste período de vida do recém-nascido, analisando a concordância entre o diagnóstico clínico e o anatomopatológico.

\section{MATERIAL E MÉTODOS}

Este estudo retrospetivo foi realizado no Serviço de Neonatologia do Centro Materno Pediátrico do Centro Hospitalar Universitário São João, unidade de nível III. Foram incluídos todos os recém-nascidos internados entre 1 de janeiro de 2008 e 31 de dezembro de 2017 que faleceram entre os zero e os sete dias de vida.

O consentimento para a realização da autópsia clínica é sistematicamente solicitado neste hospital. Após o consentimento informado dos pais, a autópsia é realizada por um patologista fetal e neonatal experiente do Serviço de Anatomia Patológica deste hospital e executada de acordo com um protocolo pré-estabelecido.

Os dados foram obtidos através da consulta do processo clínico do recém-nascido e da mãe, notas de transferência, notas de alta e relatórios de autópsia.

Foram analisadas as seguintes variáveis maternas: idade, paridade, história de abortos, morte fetal e neonatal e história clínica passada. As variáveis do recém-nascido incluem: idade gestacional, idade à admissão e no falecimento, sexo, peso ao nascimento e história obstétrica (tipo de parto e complicações obstétricas).

O diagnóstico de causa de morte clínica foi obtido através da consulta do processo clínico e das notas de alta e

Tabela 1 - Classificação de concordância entre diagnóstico ante-mortem e post-mortem*
Diagnóstico que, se tivesse sido conhecido antes da morte, provavelmente teria levado a uma alteração no tratamento que poderia ter resultado em cura ou prolongado a sobrevida.
IB
Diagnóstico com implicações significativas para futuro aconselhamento genético
II Diagnóstico que, se tivesse sido detetado antes da morte, provavelmente não levaria a mudanças no tratamento ou sobrevida porque:
A. Nenhuma terapia apropriada estava disponível no momento
B. A terapia apropriada foi dada mesmo que o diagnóstico fosse desconhecido na ocasião
C. O doente teve uma paragem cardiorrespiratória aguda que foi adequadamente controlada, mas o doente não sobreviveu para tratamento definitivo
D. O doente tem indicação clínica de "não reanimar"

III Diagnóstico que pode ou não ter sido relacionado com o processo principal de doença e foi causa contributiva de morte.

V Completa concordância entre o diagnóstico antes da morte e os achados na autópsia

*: Segundo a classificação apresentada por Brodlie et $a^{12}$ (modificada de Goldman e Kumar) 
o diagnóstico da causa de morte após a autópsia foi obtida através da análise dos relatórios anatomopatológicos. A causa de morte foi classificada utilizando a classificação de Wigglesworth, ${ }^{11}$ que é a mais usada internacionalmente e utilizada em Portugal para o registo nacional da mortalidade perinatal. Nesta classificação as quatro causas de morte neonatal principais são: anomalias congénitas; imaturidade ou parto pré-termo (prematuridade); asfixia durante o trabaIho de parto; e outras causas.

Para avaliar a concordância entre os diagnósticos clínicos e achados anatomopatológicos foi utilizada uma classificação de Goldman modificada ${ }^{12}$ (Tabela 1), que divide os achados adicionais da autópsia em major (classe I e II), que inclui diagnósticos de causa primária de morte ou doenças subjacentes e minor (classe III e IV), que inclui diagnósticos relacionados, causas associadas à causa de morte ou outras condições relevantes. Os casos foram classificados de acordo com o achado do exame necrópsico mais relevante.

Os dados foram analisados utilizando o programa SPSS da IBM Statistic, versão 25. As variáveis foram descritas com frequências absolutas e relativas para variáveis categóricas e mediana, percentil 25 (p25) e percentil 75 (p75) para variáveis contínuas. A comparação entre grupos foi realizada com o teste qui-quadrado, exato de Fisher e Mann-Whitney.

Para identificar características dos recém-nascidos e fatores clínicos associados à realização de autópsia foi efetuada a análise de regressão logística utilizando a técnica de seleção regressiva (backward stepwise). Todas as variáveis com um nível de significância de $p \leq 0,2$ na aná- lise univariada foram consideradas para o modelo final. As variáveis contínuas foram verificadas quanto à sua linearidade através do procedimento Box-Tidwell. ${ }^{13} \mathrm{~A}$ força de associação foi avaliada pelo odds ratio (OR) e seu respetivo intervalo de confiança (IC) de 95\%. Valores de $p$ inferiores a 0,05 foram considerado significativos.

O estudo foi aceite pela Comissão de Ética para a Saúde do Centro Hospitalar Universitário São João que dispensou a necessidade de consentimento informado para a realização deste estudo.

\section{RESULTADOS}

Durante o período do estudo foram internados 4026 recém-nascidos, tendo falecido 157 , o que corresponde a uma taxa de mortalidade de $3,9 \%$. Setenta e seis $(48,4 \%)$ faleceram nos primeiros sete dias de vida, $52(33,1 \%)$ nos restantes 28 dias de vida e 29 (18,5\%) após o período neonatal. Dos $128(81,5 \%)$ que faleceram no período neonatal, $59,4 \%$ faleceram no período neonatal precoce. A autópsia foi realizada a $50(65,8 \%)$ dos que faleceram na primeira semana de vida. As taxas de autópsia anuais variaram entre um máximo de $87,5 \%$ em 2011 e um mínimo de $37,5 \%$ em 2015.

As causas de morte segundo a classificação de Wigglesworth encontram-se descritas na Tabela 2.

A idade materna foi igual ou inferior a 34 anos em $57(75,0 \%)$ casos; $46(60,5 \%)$ eram primíparas e em 61 $(84,7 \%)$ não havia registo de antecedentes médicos relevantes. Em relação aos antecedentes encontrados, quatro tinham história de hipertensão arterial crónica, três de

Tabela 2 - Causas de morte dos recém-nascidos do estudo segundo classificação de Wigglesworth ${ }^{11}$

\begin{tabular}{|c|c|c|c|}
\hline Classificação & n (\%) & & $\mathbf{n}$ \\
\hline \multirow[t]{8}{*}{ Anomalias congénitas } & \multirow[t]{8}{*}{$28(36,8)$} & Doença SNC & 2 \\
\hline & & Doença cardiovascular & 6 \\
\hline & & Doença do aparelho digestivo & 1 \\
\hline & & Doença do aparelho urinário & 2 \\
\hline & & Doença do sistema musculoesquelético & 6 \\
\hline & & Anomalia cromossómica & 1 \\
\hline & & Síndrome polimalformativo não cromossómco & 3 \\
\hline & & Outra & 7 \\
\hline \multirow[t]{5}{*}{ Imaturidade ou parto pré termo } & \multirow[t]{5}{*}{$33(43,4)$} & Doença membrana hialina & 12 \\
\hline & & Infeção & 7 \\
\hline & & Enterocolite necrosante & 4 \\
\hline & & Hemorragia sistema nervoso central & 9 \\
\hline & & Outra & 1 \\
\hline \multirow[t]{2}{*}{ Asfixia durante trabalho de parto } & \multirow[t]{2}{*}{$11(14,5)$} & Descolamento placenta & 4 \\
\hline & & Outras & 7 \\
\hline \multirow[t]{4}{*}{ Outras causas não incluídas nos grupos anteriores } & \multirow[t]{4}{*}{$4(5,3)$} & Doença do sistema nervoso central & 1 \\
\hline & & Doença cardiovascular & 1 \\
\hline & & Doença metabólica & 1 \\
\hline & & Infeção bacteriana materna & 1 \\
\hline
\end{tabular}


doença tiroideia, uma de diabetes mellitus tipo 2, uma de epilepsia, uma de trombofilia hereditária e ainda um caso com antecedentes de trombose venosa profunda e tromboembolismo pulmonar. Estava documentada história de aborto em $12(15,8 \%)$ casos e dois $(2,7 \%)$ casos tinham história de morte fetal ou neonatal.

Setenta e quatro $(94,7 \%)$ gestações foram vigiadas e $62(81,6 \%)$ foram únicas. Em $50(68,5 \%)$ a gestação apresentou alguma complicação, como o diagnóstico pré-natal de malformações $(n=19)$, sinais clínicos suspeitos de corioamnionite $(n=12)$, rutura prematura ou prolongada de membranas $(n=10)$, descolamento de placenta $(n=10)$, pré-eclampsia ( $n=7)$, alteração dos fluxos da artéria umbilical $(n=7)$, restrição de crescimento intrauterino $(n=7)$, diabetes gestacional ( $n=6)$, insuficiência cervical $(n=5)$, centralização da circulação fetal $(n=2)$, ameaça de parto pré-termo com necessidade de internamento $(n=2)$, infeção genital $(n=1)$, síndrome de HELLP $(n=1)$, placenta prévia $(n=1)$, taquicardia fetal $(n=1)$, extrofia do saco amniótico $(n=1)$, discordância do crescimento fetal $(n=1)$.

Em relação aos recém-nascidos, 41 (53,9\%) eram do sexo masculino, $47(61,8 \%)$ nasceram na nossa unidade hospitalar, $64(84,2 \%)$ foram internados na UCIN nas primeiras 24 horas de vida, tendo falecido em média com dois dias de vida. A mediana de idade gestacional foi de 31 semanas, sendo $67,1 \%$ dos recém-nascidos prematuros (< 37 semanas de gestação). A mediana de peso ao nascimento foi de $1525 \mathrm{~g}$, sendo inferior a $2500 \mathrm{~g}$ em $53(69,8 \%)$

Tabela 3 - Características clínicas dos recém-nascidos, comparando os grupos com e sem realização da autópsia

\begin{tabular}{|c|c|c|c|c|}
\hline & $\begin{array}{c}\text { Total } \\
(\mathrm{n}=76)\end{array}$ & $\begin{array}{l}\text { Com autópsia } \\
\qquad(n=50)\end{array}$ & $\begin{array}{l}\text { Sem autópsia } \\
\qquad(n=26)\end{array}$ & $p$ \\
\hline $\begin{array}{l}\text { Idade falecimento (dias) } \\
\text { (mediana; p25/p75) }\end{array}$ & $\begin{array}{c}2 \\
(0 / 4)\end{array}$ & $\begin{array}{c}1 \\
(1 / 3)\end{array}$ & $\begin{array}{c}3 \\
(0 / 6)\end{array}$ & $0,119^{*}$ \\
\hline$<24$ horas $\mathrm{n}(\%)$ & $21(27,6)$ & $12(24,0)$ & $9(34,6)$ & $0,326^{* *}$ \\
\hline $\begin{array}{l}\text { Idade gestacional (semanas) } \\
\text { (mediana; } 25 / \mathrm{p} 75 \text { ) }\end{array}$ & $\begin{array}{c}31 \\
(26 / 38)\end{array}$ & $\begin{array}{c}35 \\
(28 / 38)\end{array}$ & $\begin{array}{c}27 \\
(25 / 35)\end{array}$ & $0,004^{*}$ \\
\hline Idade gestacional (semanas) n (\%) & & & & $0,013^{\star * \star}$ \\
\hline $23-28$ & $32(42,1)$ & $15(30,0)$ & $17(65,4)$ & \\
\hline $29-36$ & $19(25,0)$ & $15(30,0)$ & $4(15,4)$ & \\
\hline$\geq 37$ & $25(32,9)$ & $20(40,0)$ & $5(19,2)$ & \\
\hline $\begin{array}{l}\text { Peso ao nascimento }(\mathrm{g}) \\
\text { (mediana; } 25 / \mathrm{p} 75)\end{array}$ & $\begin{array}{c}1525 \\
(765 / 2613)\end{array}$ & $\begin{array}{c}1945 \\
(854 / 2980)\end{array}$ & $\begin{array}{c}870 \\
(665 / 2160)\end{array}$ & $0,011^{*}$ \\
\hline Peso ao nascimento $(\mathrm{g})(\mathrm{n} ; \%)$ & & & & $0,031^{* * *}$ \\
\hline$<1500$ & $37(48,7)$ & $19(38,0)$ & $18(69,2)$ & \\
\hline $1500-2499$ & $16(21,1)$ & $12(24,0)$ & $4(15,4)$ & \\
\hline$\geq 2500$ & $23(30,3)$ & $19(38,0)$ & $4(15,4)$ & \\
\hline \multicolumn{5}{|l|}{ Tipo de parto } \\
\hline Cesariana n (\%) & $41(53,9)$ & $30(60,0)$ & $11(42,3)$ & $0,155^{* *}$ \\
\hline \multicolumn{5}{|l|}{ Índice APGAR n (\%) } \\
\hline $1^{\circ}$ minuto $<7$ & $55(72,4)$ & $34(68,0)$ & $21(80,8)$ & $0,238^{* *}$ \\
\hline $5^{\circ}$ minuto $<7$ & $37(48,7)$ & $21(42,0)$ & $16(61,5)$ & $0,106^{* *}$ \\
\hline Reanimação n (\%) & $59(78,7)$ & $37(74,0)$ & $22(88,0)$ & $0,235^{\star *}$ \\
\hline Oxigénio n (\%) & $59(78,7)$ & $37(74,0)$ & $22(88,0)$ & $0,235^{* *}$ \\
\hline Tubo endotraqueal $\mathrm{n}(\%)$ & $49(65,3)$ & $29(58,0)$ & $20(80,0)$ & $0,074^{* *}$ \\
\hline Adrenalina $\mathrm{n}(\%)$ & $13(17,6)$ & $7(14,3)$ & $6(24,0)$ & $0,342^{* *}$ \\
\hline Ventilação mecânica à admissão n (\%) & $67(90,5)$ & $42(87,5)$ & $25(96,2)$ & $0,410^{* *}$ \\
\hline $\begin{array}{l}\text { Idade materna (anos) } \\
\text { (mediana; p25/p75) }\end{array}$ & $\begin{array}{c}31 \\
(27 / 35)\end{array}$ & $\begin{array}{c}32 \\
(27 / 35)\end{array}$ & $\begin{array}{c}30 \\
(26 / 33)\end{array}$ & $0,256^{*}$ \\
\hline Idade materna (anos) n (\%) & & & & $0,300^{* * *}$ \\
\hline$\leq 34$ & $57(75,0)$ & $35(70,0)$ & $22(84,6)$ & \\
\hline $35-39$ & $15(19,7)$ & $11(22,0)$ & $4(15,4)$ & \\
\hline$\geq 40$ & $4(5,3)$ & $4(8,0)$ & 0 & \\
\hline Causa clínica de morte n (\%) & & & & $0,029^{* *}$ \\
\hline Anomalias congénitas & $24(31,6)$ & $20(40,0)$ & $4(15,4)$ & \\
\hline Outras causas & $52(68,4)$ & $30(60,0)$ & $22(84,6)$ & \\
\hline
\end{tabular}

p25/p75: percentil25/percentil75; *: Teste de Mann-Whitney; **: Teste qui-quadrado; ***: Teste exato de Fisher 
recém-nascidos.

Os recém-nascidos que realizaram autópsia foram comparados com os que não a realizaram, e na análise univariada foram preditores da realização da autópsia a idade gestacional, o peso ao nascimento e anomalias congénitas como causa clínica de morte (Tabela 3). Posteriormente foi efetuada a análise multivariada que mostrou associação entre a idade gestacional e a realização da autópsia (Tabela 4), com maior frequência de autópsia nos recém-nascidos de termo.

Analisando a informação obtida pela classificação modificada de Goldman, foram encontrados achados adicionais em $62 \%$ das autópsias (Tabela 5 ).

Foram encontrados achados major (classe I e II) em $46 \%$ dos casos. Oito (16\%) casos foram classificados como classe I, sendo que em dois (4\%) casos os achados da autópsia, se conhecidos antes da morte, poderiam ter aumentado a sobrevida, e em seis $(12 \%)$ casos a autópsia revelou informações importantes para o aconselhamento genético de futuras gestações. Quinze (30\%) casos foram classificados como classe II. Nestes, os achados encontrados na autópsia provavelmente não levariam a alteração da terapêutica ou sobrevida porque: em dois casos não estava disponível tratamento adequado para a situação clínica (classe IIA), em oito casos o recém-nascido estava a receber o tratamento adequado apesar de não ter sido feito o diagnóstico da situação clínica (classe IIB), em quatro casos o recém-nascido teve uma paragem cardiorrespiratória que, apesar de tentativas de reanimação, levou ao seu falecimento precoce, não tendo havido tempo para chegar ao diagnóstico (classe IIC) e num caso, o recém-nascido tinha indicação para não ser reanimado (classe IID).

Foram encontrados achados minor (classe III e IV) em $16 \%$ dos casos. Quatro (8\%) casos foram classificados como classe III e quatro (8\%) casos como classe IV.

Em 19 (38\%) casos, houve concordância total entre o diagnóstico clínico e o anatomopatológico (Tabela 5).

O diagnóstico mais frequentemente não identificado clinicamente foi pneumonia $(n=8)$, embora em todos os casos o doente estivesse sob antibioterapia adequada por suspeita de outra infeção.

Por outro lado, o diagnóstico mais frequentemente sobrediagnosticado clinicamente foi a doença das membranas hialinas, com cinco casos em que esse diagnóstico não foi confirmado pela autópsia.

Os casos classificados como classe I e II estão descri-

Tabela 4 - Determinantes da realização de autópsia

\begin{tabular}{lccc}
\hline Variáveis & OR $^{*}$ & IC $95 \%$ & $p$ \\
\hline $\begin{array}{l}\text { Idade gestacional } \\
\text { (semanas) }\end{array}$ & & & \\
$23-28$ & - & & \\
$29-36$ & 4,77 & $1,17-17,68$ & $\mathbf{0 , 0 2 9}$ \\
$\geq 37$ & 5,78 & $1,33-16,17$ & $\mathbf{0 , 0 1 6}$ \\
APGAR $\mathbf{5}^{\circ}<\mathbf{7}$ & 3,66 & $0,97-8,28$ & 0,056 \\
\hline
\end{tabular}

tos na Tabela 6.

\section{DISCUSSÃO}

Dos 157 recém-nascidos que faleceram durante o período de estudo, 128 (81,5\%) faleceram no período neonatal, tendo a maioria desses falecido no período neonatal precoce $(59,4 \%)$.

A principal causa de morte foi devido a complicações relacionadas com prematuridade, tendo esta ocorrido em $33(43,4 \%)$ casos. Apesar de ser a principal causa de morte neonatal, os óbitos de recém-nascidos prematuros têm vindo a diminuir nos últimos 20 anos tanto na Europa como na América do Norte. ${ }^{2}$

As anomalias congénitas estão na origem de cinco a $38 \%$ das mortes neonatais. No nosso estudo encontramos em $36,8 \%$ (28 casos). Estas parecem ter um maior papel na mortalidade neonatal precoce em países desenvolvidos. Nestes países tem-se assistido à diminuição da mortalidade por outras causas, designadamente infeciosas, devido à adequada vigilância da gravidez. ${ }^{2}$

A asfixia no período perinatal é uma das principais causas de morte precoce em todo mundo, sendo responsável por $7,1 \%$ das mortes neonatais precoces em países desenvolvidos. ${ }^{2}$ O nosso estudo revelou uma maior percentagem de mortes neonatais precoces associadas à asfixia durante o trabalho de parto $(14,5 \%)$. Esta diferença poderá estar relacionada com o facto deste hospital ser um centro de referência para hipotermia induzida.

A importância da autópsia neonatal já foi discutida e demonstrada em alguns estudos realizados em unidades de cuidados intensivos neonatais de diferentes países, tendo a taxa de autópsia neonatal variado entre 39\% e $82 \% .{ }^{14}$ No entanto, estudos realizados avaliando apenas a importância da autópsia no período neonatal precoce são escassos. A morte neonatal precoce representa uma percentagem importante das mortes ocorridas no período neonatal, confirmando que a primeira semana de vida é a mais crítica para o recém-nascido. ${ }^{2} \mathrm{~A}$ maioria dos que morrem neste período de vida são prematuros, sendo a causa de morte muitas vezes considerada relacionada com as complicações da

Tabela 5 - Distribuição dos achados de acordo com a classificação de Goldman modificada

\begin{tabular}{lcc}
\hline \multicolumn{2}{c}{ Classe } & $\mathbf{n}(\%)$ \\
\hline I & & $8(16)$ \\
& A & 2 \\
& B & 6 \\
II & & $15(30)$ \\
& A & 2 \\
& B & 8 \\
& C & 4 \\
& D & 1 \\
III & & $4(8)$ \\
IV & & $4(8)$ \\
V & & $19(38)$ \\
\hline
\end{tabular}


Tabela 6 - Descrição dos casos classificados como classe I e II

\begin{tabular}{|c|c|c|}
\hline & Diagnóstico clínico & Diagnóstico anatomopatológico \\
\hline \multicolumn{3}{|l|}{ Classe IA } \\
\hline Caso 1 & Prematuridade com restrição de crescimento & Derrame pericárdico (quase-tamponamento) \\
\hline Caso 2 & Hérnia diafragmática congénita & Coartação da aorta; Hérnia diafragmática congénita. \\
\hline \multicolumn{3}{|l|}{ Classe IB } \\
\hline Caso 1 & Síndrome junção pielo-uretral bilateral & $\begin{array}{l}\text { Displasia dos capilares alveolares; pneumonia bilateral; } \\
\text { Síndrome junção pielo-uretral bilateral. }\end{array}$ \\
\hline Caso 2 & Insuficiência hepática de etiologia desconhecida & Hemocromatose congénita; Pneumonia bilateral congénita. \\
\hline Caso 3 & Hérnia diafragmática congénita & $\begin{array}{l}\text { Achados ósseos sugestivos de osteocondroplasia; Hérnia } \\
\text { diafragmática congénita. }\end{array}$ \\
\hline Caso 4 & Cardiopatia congénita & $\begin{array}{l}\text { Linfangiectasia pulmonar; Pneumonia bilateral; Cardiopatia } \\
\text { congénita. }\end{array}$ \\
\hline Caso 5 & Quilotorax congénito no contexto de hidropsia fetal & Displasia linfática; Quilotorax congénito. \\
\hline Caso 6 & $\begin{array}{l}\text { Provável malformação vascular pulmonar associada } \\
\text { a hipertensão pulmonar }\end{array}$ & Linfangiectasia pulmonar congénita. \\
\hline \multicolumn{3}{|l|}{ Classe IIA } \\
\hline Caso 1 & Cardiopatia congénita (provável Ebstein) & $\begin{array}{l}\text { Cardiopatia congénita não Ebstein; Miocardio não compactado } \\
\text { com disfunção da microcirculação coronária e atingimento } \\
\text { válvula tricúspide. }\end{array}$ \\
\hline Caso 2 & Cardiopatia congénita provável & Cardiopatia complexa - Isomerismo direito. \\
\hline \multicolumn{3}{|l|}{ Classe IIB } \\
\hline Caso 1 & Hérnia diafragmática congénita & Hérnia diafragmática congénita; Pneumonia aguda direita. \\
\hline Caso 2 & Doença membrana hialina & Síndrome polimalformativo. \\
\hline Caso 3 & Entercolite Necrosante Perfurada (ENP) & $\begin{array}{l}\text { ENP com choque séptico e peritonite aguda complicada com } \\
\text { coagulação intravascular disseminada (CID) e isquemia cerebral; } \\
\text { Meningite incipiente; Enfarte. }\end{array}$ \\
\hline Caso 4 & Doença membrana hialina & $\begin{array}{l}\text { Asfixia durante trabalho de parto com descolamento da placenta; } \\
\text { Doença membranas hialinas incipiente. }\end{array}$ \\
\hline Caso 5 & Doença membrana hialina grave & $\begin{array}{l}\text { Pneumonia congénita com imaturidade pulmonar; Enfisema } \\
\text { intersticial pulmonar. }\end{array}$ \\
\hline Caso 6 & Síndrome de Edwards & Síndrome de Edwards; Pneumonia bilateral. \\
\hline Caso 7 & Doença membrana hialina & Pneumonia bilateral; Meningite. \\
\hline Caso 8 & Doença membrana hialina & $\begin{array}{l}\text { Pneumonia necrosante; Pneumotorax à direita; Doença } \\
\text { membranas hialinas. }\end{array}$ \\
\hline \multicolumn{3}{|l|}{ Classe IIC } \\
\hline Caso 1 & Cardiopatia congénita provável & $\begin{array}{l}\text { Cardiopatia congénita - Transposição completa das grandes } \\
\text { artérias. }\end{array}$ \\
\hline Caso 2 & Doença membrana hialina & $\begin{array}{l}\text { Pneumonite intersticial com hipoplasia pulmonar com } \\
\text { imaturidade relativa multifocal. }\end{array}$ \\
\hline Caso 3 & Coagulação intravascular disseminada (CID) & $\begin{array}{l}\text { Neoplasia congénita para-vertebral (provável neuroblastoma); } \\
\text { CID. }\end{array}$ \\
\hline Caso 4 & Discrasia sanguínea grave & Neuroblastoma congénito com metástases hepáticas. \\
\hline \multicolumn{3}{|l|}{ Classe IID } \\
\hline Caso 1 & $\begin{array}{l}\text { Síndrome aspiração meconial com hipertensão } \\
\text { pulmonar grave refratária; Sepsis neonatal precoce. } \\
\text { Indicação clínica de 'não reanimar'. }\end{array}$ & $\begin{array}{l}\text { Sepsis neonatal; Pneumonia bilateral; Encefalite aguda; } \\
\text { Pneumotorax. }\end{array}$ \\
\hline
\end{tabular}

prematuridade, o que faz com que a autópsia possa ser considerada desnecessária. ${ }^{15}$ No entanto, a autópsia perinatal continua a ser o método mais útil para compreender a causa e os mecanismos da morte, alterando o diagnóstico clínico em $35 \%$ a $75 \%$ dos casos. ${ }^{5}$

No nosso estudo, a taxa de autópsia neonatal precoce foi de $65,8 \%$, sendo superior à encontrada por outros autores $(47,9 \%) .{ }^{15} \mathrm{O}$ exame necrópsico foi realizado com maior frequência em recém-nascidos com maior idade gestacional. Estudos que também incluíram óbitos após os sete dias de vida mostraram resultados semelhantes. . $^{8,12,16}$ Estes resultados podem ser explicados pelo facto de a realização da autópsia ser menos encorajada na prematuridade extrema. ${ }^{8,15}$ Para além disso, está descrito que o peso 
ao nascimento e a presença de malformações congénitas também influenciam na decisão de realização da autópsia neonatal. Em relação ao peso ao nascimento, está descrito que a autópsia neonatal é realizada mais frequentemente em recém-nascidos com maior peso ao nascimento. ${ }^{16}$ Nesta amostra, a maioria dos recém-nascidos que não realizaram autópsia eram recém-nascidos de muito baixo peso ao nascimento (Tabela 3 ), à semelhança do referido na literatura. ${ }^{15} \mathrm{Em}$ relação à presença de malformações congénitas, existem resultados contraditórios na literatura. Alguns autores mostraram que a autópsia foi realizada com maior frequência em recém-nascidos que apresentavam malformações congénitas, ${ }^{16}$ enquanto noutros esta foi realizada maioritariamente nos recém-nascidos que não as apresentavam. ${ }^{8}$ Apesar de não ser significativo na análise multivariada, a autópsia foi realizada mais frequentemente em recém-nascidos em que a causa de morte foi atribuída a anomalias congénitas. As anomalias congénitas são entendidas como manifestações óbvias de doenças que podem ser causa de morte, podendo a realização de autópsia ser menos encorajada nestes casos. ${ }^{8}$ No entanto, mesmo com o diagnóstico clínico de malformação congénita a realização da autópsia é muito útil, permitindo um diagnóstico clínico mais correto. ${ }^{3}$

Foram encontrados achados adicionais na autópsia (classe I-IV) em $62 \%$ dos casos, em que $46 \%$ foram achados major e em $16 \%$ foram achados minor. Os achados encontrados, se conhecidos antes da morte, provavelmente levariam a alteração do prognóstico em $4 \%$ dos casos (classe IA). Em 12\% dos casos os achados encontrados na autópsia foram relevantes para o aconselhamento genético de futuras gestações (classe IB), o que vem reforçar a sua importância.

O achado adicional mais frequente no exame anatomopatológico foi pneumonia, o que está de acordo com outras publicações. ${ }^{4,7}$

Observou-se também uma sobrestimação do diagnóstico clínico de doença das membranas hialinas. Em cinco casos que tiveram o diagnóstico clínico desta doença, a autópsia não revelou achados compatíveis com esta condição clínica. No entanto, todos os doentes realizaram terapêutica com surfactante exógeno, o que poderia justificar a sua ausência.

Um estudo prévio realizado na nossa UCIN em 2010 e que teve como objetivo avaliar a concordância entre diagnósticos ante-mortem e post-mortem em todos os recém-nascidos admitidos na unidade, usando a mesma classificação de Goldman modificada demonstrou resultados semelhantes com os do presente estudo, revelando uma percentagem de achados adicionais de 66\% (53\% achados major e $13 \%$ achados minor) e tendo em $9,4 \%$ casos informação relevante para aconselhamento genético. ${ }^{4}$

A revisão sistemática de Custer et $a /^{14}$ analisou seis publicações que estudaram a presença de erros de diagnóstico em unidades de cuidados intensivos neonatais de diferentes países, de acordo com classificação de Goldman. Achados adicionais major estavam presentes em 19,2\% dos casos (classe I, 3,7\%; classe II, 15,5\%). Achados classe III foram apenas relatados em cinco publicações e foram identificados em $37,2 \%$ dos casos. Apenas três estudos relataram diagnósticos da classe IV, estando estes presentes em 15,6\% dos casos. Comparativamente, a nossa casuística apresentou uma maior percentagem de achados major (46\%). Isto pode ser explicado pelo facto desta revisão sistemática incluir recém-nascidos que faleceram com mais de sete dias de vida, tendo maior probabilidade de ter um quadro clínico mais esclarecido, comparativamente com os que faleceram na primeira semana de vida, apesar de tal facto não se ter verificado aquando da comparação do nosso estudo com o realizado na mesma instituição em 2010. ${ }^{4}$ A subjetividade da classificação dos casos segundo a classificação de Goldman é uma possibilidade que não podemos excluir. Em relação aos achados minor, o nosso estudo apresentou um menor número de achados (classe III foi de $8 \%$ e classe IV foi de $8 \%$ ). Esta situação pode ser explicada pelo facto de alguns estudos incluídos na revisão relatarem vários achados por doente, incluindo o mesmo caso em diferentes classes, o que resulta numa percentagem ligeiramente maior. Para além disso, já foi descrito que os achados minor são encontrados mais frequentemente em recém-nascidos que faleceram com mais dias de vida. ${ }^{3}$ Uma vez que neste estudo apenas foram incluídos os recém-nascidos que faleceram na primeira semana de vida, este facto também pode explicar o menor número de achados minor.

A autópsia é particularmente importante no período neonatal porque, para além de fornecer informações objetivas sobre a causa de morte, permite detetar condições importantes para aconselhamento genético de futuras gestações. ${ }^{6}$ Neste estudo a autópsia revelou informação relevante para aconselhamento genético em $12 \%$ dos casos. A percentagem desses achados em outros estudos variou entre $2,4 \%$ a $26 \%$, tendo o estudo anterior realizado na nossa UCIN mostrado uma percentagem de 9,4\%.4,6,12 Estes resultados reforçam ainda mais a importância da realização da autópsia no período neonatal precoce.

Este estudo tem algumas limitações. É retrospetivo, com uma amostra reduzida, e foi realizado num único centro, o que limita a generalização dos resultados. O viés de seleção não pode ser esquecido, uma vez que os pais de recém-nascidos com um diagnóstico clínico incerto provavelmente deram o seu consentimento para a realização da autópsia mais facilmente.

Os pontos fortes a realçar neste estudo são o facto de ser realizado num serviço de neonatologia de um hospital de nível III, deste ser um centro de referência para patologia neonatal cardíaca, cirúrgica e neurológica e ter uma elevada taxa de autópsia (65,8\%).

A percentagem de diagnósticos adicionais encontrada em estudos recentes e no presente estudo demonstram que, apesar da melhoria dos métodos de diagnóstico nos últimos anos, a autópsia é ainda importante para esclarecer a causa de morte e dar a conhecer outras condições não diagnosticadas previamente, mesmo na presença de 
fatores óbvios de causa de morte, como prematuridade extrema ou anomalias congénitas. Uma vez que não é possível prever em que recém-nascidos a autópsia revelará informações importantes, ${ }^{8}$ esta deverá ser proposta a todos os pais após a morte neonatal precoce.

\section{CONCLUSÃO}

O resultado da autópsia é fundamental no esclarecimento das causas de morte neonatal precoce, mesmo nos casos em que o diagnóstico clínico justifica a causa de morte. Os achados adicionais encontrados no exame anatomopatológico são cruciais para um diagnóstico correto e muitas vezes permitem o aconselhamento genético. Assim, a autópsia deve ser proposta a todos os pais após a morte neonatal precoce, explicando a sua importância e sublinhando como esta pode ser relevante em gestações futuras.

\section{REFERÊNCIAS}

1. UNIGME. Levels \& trends in child mortality: report 2018, estimates developed by the United Nations Inter-agency Group for Child Mortality Estimation. New York: UNICEF; 2018.

2. Lehtonen L, Gimeno A, Parra-Llorca A, Vento M. Early neonatal death: a challenge worldwide. Semin Fetal Neonatal Med. 2017;22:153-60.

3. de Sevaux JLH, Nikkels PGJ, Lequin MH, Groenendaal F. The Value of Autopsy in Neonates in the $21^{\text {st }}$ Century. Neonatology. 2019;115:89-93.

4. Costa S, Rodrigues M, Centeno MJ, Martins A, Vilan A, Brandão O, et al. Diagnosis and cause of death in a neonatal intensive care unit--how important is autopsy? J Matern Fetal Neonatal Med. 2011;24:760-3.

5. Judge-Kronis L, Hutchinson JC, Sebire NJ, Arthurs OJ. Consent for paediatric and perinatal postmortem investigations: implications of less invasive autopsy. J Forensic Radiol Imaging. 2016;4:7-11.

6. Tamanaha F, Fuksman R, Pedraza A, Prudent L. What is the value of neonatal autopsy? Pathological and clinical correlation in 135 cases. Arch Argent Pediatr. 2017;115:490-2.

7. Feria-Kaiser C, Furuya ME, Vargas MH, Rodriguez A, Cantu MA. Main diagnosis and cause of death in a neonatal intensive care unit: do clinicians and pathologists agree? Acta Paediatr. 2002;91:453-8.

8. Swinton $\mathrm{CH}$, Weiner J, Okah FA. The neonatal autopsy: can it be revived? Am J Perinatol. 2013;30:739-44.

9. Leadbetter KZ, Vesoulis ZA, White FV, Schmidt RE, Khanna G, Shimony

\section{PROTECÇÃO DE PESSOAS E ANIMAIS}

Os autores declaram que os procedimentos seguidos estavam de acordo com os regulamentos estabelecidos pelos responsáveis da Comissão de Investigação Clínica e Ética e de acordo com a Declaração de Helsínquia da Associação Médica Mundial.

\section{CONFIDENCIALIDADE DOS DADOS}

Os autores declaram ter seguido os protocolos do seu centro de trabalho acerca da publicação de dados.

\section{CONFLITOS DE INTERESSE}

Os autores declaram não ter conflitos de interesse relacionados com o presente trabalho.

\section{FONTES DE FINANCIAMENTO}

Este projeto não recebeu qualquer apoio financeiro.

JS, et al. The role of post-mortem MRI in the neonatal intensive care unit. J Perinatol. 2017;37:98-103.

10. Celiloglu OS, Celiloglu C, Kurnaz E, Ozdemir R, Karadag A. Diagnostic contribution of postmortem needle biopsies in neonates. Turk Patoloji Derg. 2013;29:122-6.

11. Wigglesworth JS. Monitoring perinatal mortality. A pathophysiological approach. Lancet. 1980;2:684-6.

12. Brodlie M, Laing IA, Keeling JW, McKenzie KJ. Ten years of neonatal autopsies in tertiary referral centre: retrospective study. BMJ. 2002;324:761-3.

13. Hosmer D, Lemeshow S. Regressão logística aplicada. $2^{\mathrm{a}}$ ed. Nova lorque: JohnWiley \& Sons; 2000.

14. Custer JW, Winters BD, Goode V, Robinson KA, Yang T, Pronovost PJ, et al. Diagnostic errors in the pediatric and neonatal ICU: a systematic review. Pediatr Crit Care Med. 2015;16:29-36.

15. Bishop KL, Dupuis C, Nanton P, Clarke K, Bolt C, Chin-See C. Perinatal autopsy rates at the University Hospital of the West Indies: 2002-2008. West Indian Med J. 2013;62:35-8.

16. Pavon Delgado A, Salinas Martin MV, Losada Martinez A, FontillonAlberdi M, Nunez Solis JM, Zamarriego Zubizarreta C. Valor de la autopsia en una unidad de cuidados intensivos neonatológicos. An Pediatr. 2008;69:124-8. 